\title{
Mortalidade por câncer entre pintores brasileiros das regiões Sul e Sudeste do Brasil
} Cancer mortality among Brazilian painters of the South
and Southeast Region of Brazil Aline de Souza Espindola Santos ${ }^{1}$, Amanda Alzira Friaes Martins ${ }^{2}$,
Jaime Silva de Lima², Armando Meyer ${ }^{1}$

\begin{abstract}
Resumo
Como resultado de sua atividade profissional, pintores entram regularmente em contato com substâncias genotóxicas e carcinogênicas presentes em tintas, vernizes e massa corrida. Com isso, eles podem apresentar risco elevado para diversas doenças, dentre elas alguns cânceres. Este estudo teve por objetivo comparar a mortalidade por cânceres específicos entre pintores e a população geral nas regiões Sudeste e Sul do Brasil no período de 1996 a 2013. Os dados de morte por câncer foram obtidos por meio do Sistema de Informação sobre Mortalidade (SIM), de acordo com a 10ª revisão da Classificação Internacional de Doenças (CID-10). Para determinar os riscos de morte por cânceres específicos, foi calculada a razão de chance de mortalidade (RCM), estratificada por faixa etária (25-44 anos e 45-64 anos) e por região. Pintores mais velhos da região Sul e Sudeste apresentaram maiores chances de morte para câncer de orofaringe $(R C M=1,73$; IC95\%:1,51-1,98), hipofaringe ( $R C M=1,56$; IC95\%:1,20-2,03) e laringe

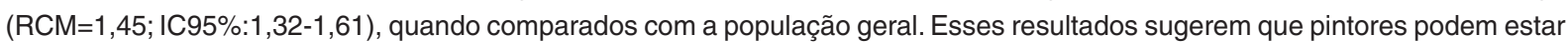
sob maior risco de adoecer e morrer por cânceres específicos como os do trato respiratório superior.
\end{abstract}

Palavras-chave: exposição ocupacional; pintores; mortalidade; câncer.

\begin{abstract}
As a result of their professional activity, painters come in regular contact with significant amounts of genotoxic and carcinogenic substances and may have increased risk for various diseases, including some cancers. This study aimed to compare the mortality by specific cancers among painters and the general population from the South and Southeast regions of Brazil from 1996 to 2013. Data on cancer deaths were obtained from the Brazilian Mortality Information System, according to ICD-10. To determine the risk of death from specific cancers, we calculated the mortality odds ratio (MOR), stratified by age (25-44 years and 45-64 years) and region. Older painters of the South and Southeast regions had a higher chance to die by oropharyngeal (MOR=1.73; $95 \%$ $\mathrm{Cl} 1.51$ to 1.98 ), hypopharynx ( $\mathrm{MOR}=1.56 ; 95 \% \mathrm{Cl} 1.20-2.03$ ) and larynx cancer ( $\mathrm{MOR}=1.45 ; 95 \% \mathrm{Cl} 1.32$ to 1.61 ) when compared to the general population. These results suggest that painters may be at greater risk of becoming ill and dying from specific cancers from the upper respiratory tract.
\end{abstract}

Keywords: occupational exposure; painters; mortality; cancer.

\footnotetext{
${ }^{1}$ Instituto de Estudos em Saúde Coletiva, Universidade Federal do Rio de Janeiro (UFRJ) - Rio de Janeiro (RJ), Brasil. ${ }^{2}$ Departamento de Bioquímica, Universidade Federal do Estado do Rio de Janeiro (UNIRIO) - Rio de Janeiro (RJ), Brasil. Trabalho realizado no Instituto de Estudos em Saúde Coletiva (IESC), Universidade Federal do Rio de Janeiro (UFRJ) - Rio de Janeiro (RJ) Brasil. Endereço para correspondência: Aline de Souza Espindola Santos - Instituto de Estudos em Saúde Coletiva, Avenida Horácio Macedo, s/n - Cidade Universitária - CEP: 21941-598 - Rio de Janeiro (RJ), Brasil - Email: esp.aline@gmail.com

Fonte de financiamento: nenhuma.

Conflito de interesses: nada a declarar.
} 


\section{INTRODUÇÃO}

Em 1989, a Agência Internacional para Pesquisa em Câncer (IARC) classificou como cancerígena para humanos (grupo 1A) a exposição ocupacional como pintor, baseada nas evidências do aumento de risco para câncer de pulmão em estudos epidemiológicos de coorte, caso-controle e metanálise, em sua maioria, ajustados para tabagismo ${ }^{1-3}$. Embora essa classificação não inclua outras neoplasias, estudos epidemiológicos sugerem associações positivas entre pintores e o desenvolvimento de cânceres de bexiga, cavidade oral, faringe, laringe e testículos ${ }^{4-7}$.

No Brasil, somente dois estudos de caso-controle ocupacionais realizados em São Paulo avaliaram a associação entre pintores e desenvolvimento de câncer. No primeiro, Wünsch-Filho et al. ${ }^{8}$ não observaram associação entre pintores com mais de dez anos de profissão e câncer de pulmão, com ou sem ajuste para tabagismo. Já Andreotti et al. ${ }^{9}$ avaliaram o risco para câncer de cavidade oral e de orofaringe combinado para diferentes ocupações. Os resultados para pintores, ajustados para fumo e bebida alcoólica, não apresentaram aumento significativo do risco para os cânceres estudados.

A exposição ocupacional em pintores pelas vias inalatória e dérmica está relacionada à volatilização de solventes durante as etapas de mistura, aplicação e transporte de tintas, massa corrida, removedores ou vernizes e a liberação de poeira contendo pigmentos, resinas e aditivos durante o lixamento de superfícies metálicas, de madeira e cimento ${ }^{3}$. Embora a inalação ou o contato dérmico sejam considerados os de maior relevância toxicológica, a via oral pode estar associada a exposições ocasionais por meio da ingestão de alimentos e bebidas no ambiente de trabalho ${ }^{3}$.

Alguns aspectos na ocupação de pintor podem intensificar a exposição a agentes químicos. Grande parte desses profissionais trabalha como autônomo e possui baixa escolaridade, característica socioeconômica importante para a compreensão dos rótulos e da toxicidade dos produtos manipulados ${ }^{10,11}$. Adicionalmente, a deficiente capacitação e a atualização profissional estão associadas à reduzida percepção dos riscos ocupacionais, sobretudo o risco químico e a baixa adesão aos equipamentos de proteção individual ${ }^{12,13}$.

O risco químico nessa atividade profissional está associado a uma variedade de substâncias genotóxicas, mutagênicas e carcinogênicas ${ }^{3}$. Do grupo 1A (cancerígenos em humanos) estão incluídos pigmentos orgânicos (2-naftilamina, 4-aminobifenil e benzidina), inorgânicos (compostos contendo cromo VI, níquel e o cádmio), solventes como o benzeno, alcatrão de hulha e formaldeído, e agentes anti-incrustantes derivados de arsênio. Substâncias do grupo 2A (provável carcinógeno para humanos) também estão presentes no processo de trabalho de pintores, como bifenilas policloradas e o tricloroetileno ${ }^{3,14,15}$.
Estudos de biomarcadores de efeitos genotóxicos têm sugerido que pintores apresentam maiores frequências de quebras de DNA, aberrações cromossômicas e micronúcleo quando comparados com os controles ${ }^{16-18}$. No Brasil, Silva e Mello (1996) observaram um aumento na frequência de aneuploidias e deleções cromossômicas em linfócitos de pintores de automóveis brasilienses em relação ao grupo controle, independentemente do hábito de fumar ${ }^{19}$. Em um estudo conduzido por Oliveira ${ }^{20}$ em Santa Catarina, trabalhadores de uma fábrica de tintas apresentaram um maior número de alterações no DNA de leucócitos e células epiteliais bucais em relação ao controle.

A presença de substâncias tóxicas em tintas e a aparente vulnerabilidade de profissionais expostos a essas substâncias evidenciam a necessidade de se avaliar os possíveis efeitos dessa exposição sobre a saúde dos pintores no Brasil. Sendo assim, o objetivo deste trabalho foi avaliar o perfil de mortalidade por neoplasias do trato respiratório (orofaringe, hipofaringe, laringe, pulmão), esôfago e testículos em pintores do sexo masculino, nas regiões Sudeste e Sul, no período de 1996 a 2013, e compará-lo ao da população geral masculina no Brasil.

\section{METODOLOGIA}

Os dados de mortalidade entre pintores e a população geral do sexo masculino foram coletados no Sistema de Informação sobre Mortalidade (SIM), utilizando a 10a revisão da Classificação Internacional de Doenças (CID-10). A busca pelos dados de morte foi feita em dois períodos (1996-2005) e (2006-2013), que estão associados a atualizações na Classificação Brasileira de Ocupações (CBO) no SIM. No primeiro período, o dado de morte em pintores foi obtido utilizando os seguintes códigos CBO (1994) de ocupação: 16100 (pintor artístico), 89500 (pintor de cerâmica), 93000 (pintor sem especificação), 93100 (pintor de construções cênicas, edifícios, embarcações, estruturas metálicas, obras, industrial), 93900 (pintor de aviões, de imersão-veículos, letreiros, manutenção, móveis, rótulos, veículos, por imersão). Para os anos de 2006-2013, foram utilizados os códigos CBO (2002): 716610 (pintor de obras), 723310 (pintor a pincel e rolo, exceto obras e estruturas), 723315 (pintor de estruturas metálicas), 723320 (pintor de veículos-fabricação), 723325 (pintor por imersão), 723330 (pintor, a pistola, exceto obras e estruturas metálicas), 752430 (pintor de cerâmica, a pincel), 768625 (pintor de letreiros), 991315 (pintor de veículos-reparação).

Inicialmente, foram avaliadas a distribuição de frequências de todas as neoplasias em relação ao total de óbitos entre pintores brasileiros e a população geral do sexo masculino por faixas etárias (25-44 e 45-64 anos), escolaridade (nenhuma, 1-7 anos, $\geq 8$ anos) e região de residência. Uma vez que as regiões Sul e Sudeste apresentam maior completude do preenchimento dos dados do SIM e cobertura dos óbitos acima de $95 \%$ em relação às demais, nossas análises se restringiram geograficamente 
a essas regiões ${ }^{21,22}$. A seguir, foi analisada a distribuição de frequência de neoplasias selecionadas (câncer de orofaringe, CID10:10; hipofaringe, CID10:13; esôfago, CID10:15; laringe, CID10:32; pulmão, CID10:34; testículos, CID10:62) em relação ao total de óbitos por neoplasias das regiões Sul e Sudeste no período de 1996 a 2013. O teste do qui-quadrado foi utilizado com nível de significância de $\mathrm{p}<0,05$ para comparar diferenças de proporções das covariáveis estudadas.

Neste estudo, a medida de associação utilizada foi a "Mortality Odds Ratio" ou razão de chances de mortalidade (RCM) proposta por Miettinen e Wang ${ }^{23}$. Para esta análise, foi necessário separar os dados de morte ou óbitos em dois grupos, a saber: o primeiro com todos os óbitos por neoplasias selecionadas e o segundo formado pelas demais causas de morte. A partir dessa separação, foram calculadas as chances de morte em pintores e na população geral, ou seja, o número de óbitos de uma neoplasia específica ou todas as neoplasias pelas demais causas de cada grupo. O cálculo da RCM baseou-se na divisão das chances entre os grupos comparados. O programa estatístico para realização das análises foi o WINPEPI, versão 11.43, com adoção do nível de significância estatística de 95\%.

A utilização de dados secundários de domínio público para a condução deste estudo não requereu aprovação pelo Comitê de Ética em Pesquisa (CEP), conforme recomendações do Conselho Nacional de Saúde em sua Resolução CNS no 466/2012.

\section{RESULTADOS}

Foi realizado um estudo epidemiológico ecológico que utilizou os dados de óbitos do SIM das regiões Sul e Sudeste do Brasil como unidade de análise. A frequência de óbitos masculinos por todas as neoplasias na população geral foi maior que aquela observada entre pintores (Tabela 1). Em relação à idade, verificou-se que a maior parte dos óbitos por neoplasias ocorreram na população geral entre os mais velhos (45-64 anos: $85,3 \%)$. No que se refere à escolaridade, $70,8 \%$ dos óbitos eram de pintores que apresentaram de um a sete anos de estudo, enquanto que, na população geral, $32,1 \%$ possuíam maior grau de instrução (oito anos ou mais). A variável escolaridade reflete a incompletude dos dados na população estudada, uma vez que, do total dos dados analisados, somente em $44,1 \%$ dos óbitos em pintores existiam informações relacionadas ao grau de instrução. Na população geral, essa informação foi obtida em $61,3 \%$ dos dados avaliados. Na estratificação por região, foi observada uma maior frequência por neoplasias em pintores $(73,2 \%)$ na região Sudeste.

Na Tabela 2, podemos observar que, em relação ao total de óbitos masculinos por neoplasias, $39,1 \%$ foram atribuídas às neoplasias selecionadas (orofaringe, hipofaringe, esôfago, laringe, pulmão, testículos) em pintores contra $33,3 \%$ na população geral. Entre os cânceres selecionados, o de pulmão foi a maior causa de morte na população geral $(45,7 \%)$, enquanto em pintores, foram o de laringe (19,2\%), de orofaringe (10,9\%) e de hipofaringe (2,8\%). Não foram observadas diferenças na porcentagem de óbitos para o câncer de esôfago e testículos entre os grupos estudados.

Em relação à RCM, para os dados não estratificados por faixa etária (total), observamos um aumento do risco por câncer de orofaringe (RCM=1,48; IC95\%:1,30-1,68), de hipofaringe ( $\mathrm{RCM}=1,30$; IC95\%:1,01-1,67) e de laringe ( $\mathrm{RCM}=1,32$; IC95\%:1,12-1,36) entre pintores homens, quando comparados à população geral (Tabela 3). Quando os dados foram estratificados, o grupo de pintores mais jovens (25-44 anos) não apresentou aumento no risco de morte para nenhum dos cânceres estudados. Já entre os pintores mais velhos, verificamos aumento no risco para câncer de orofaringe (RCM=1,73; IC95\%:1,51-1,98), de hipofaringe ( $\mathrm{RCM}=1,56$; IC95\%:1,20-2,03), de laringe $(\mathrm{RCM}=1,45$;

Tabela 1. Distribuição dos óbitos na população estudada segundo faixa etária e escolaridade das regiões Sudeste e Sul do Brasil, de 1996-2013

\begin{tabular}{|c|c|c|c|c|c|c|}
\hline \multirow{2}{*}{ 1996-2013 } & \multicolumn{2}{|c|}{ Pintores } & \multicolumn{2}{|c|}{ População geral } & \multirow{2}{*}{$\chi^{2}$} & \multirow{2}{*}{$\mathbf{p}$} \\
\hline & $\mathbf{N}$ & $\%$ & $\mathbf{N}$ & $\%$ & & \\
\hline Total & 45535 & 100 & 2864599 & 100 & \multirow{2}{*}{148,8} & \multirow{2}{*}{$0,0 \mathrm{C}$} \\
\hline Neoplasias & 5689 & 12,5 & 425282 & 14,8 & & \\
\hline \multicolumn{7}{|c|}{ Faixas etárias } \\
\hline 25-44 anos & 989 & 17,4 & 62455 & 14,7 & \multirow{3}{*}{32,5} & \multirow{3}{*}{0,00} \\
\hline 45-64 anos & 4700 & 82,6 & 362827 & 85,3 & & \\
\hline Total & 5689 & 100 & 425282 & 100 & & \\
\hline \multicolumn{7}{|l|}{ Educação } \\
\hline Nenhuma & 192 & 7,5 & 22066 & 8,5 & \multirow{4}{*}{142,3} & \multirow{4}{*}{0,00} \\
\hline 1-7 anos & 1801 & 70,8 & 155032 & 59,5 & & \\
\hline$\geq 8$ anos & 551 & 21,7 & 83637 & 32,1 & & \\
\hline Total & 2544 & 44,1 & 260735 & 61,3 & & \\
\hline \multicolumn{7}{|l|}{ Região } \\
\hline Sudeste & 4164 & 73,2 & 297332 & 69,9 & \multirow{3}{*}{28,7} & \multirow{3}{*}{0,00} \\
\hline Sul & 1525 & 26,8 & 127950 & 30,1 & & \\
\hline Total & 5689 & 100 & 425282 & 100 & & \\
\hline
\end{tabular}

Tabela 2. Distribuição de óbitos por neoplasias e por neoplasias selecionadas em pintores das regiões Sudeste e Sul do Brasil, de 1996-2013

\begin{tabular}{|c|c|c|c|c|c|c|c|}
\hline & \multicolumn{2}{|c|}{ Pintores } & \multicolumn{2}{|c|}{ População geral } & \multirow{2}{*}{$\chi^{2}$} & \multirow{2}{*}{$\mathbf{p}$} \\
\hline & & $\mathbf{N}$ & $\%$ & $\mathbf{N}$ & $\%$ & & \\
\hline \multicolumn{2}{|c|}{ Neoplasias } & 5689 & 100,0 & 425282 & 100 & \multirow{3}{*}{41,0} & \multirow{3}{*}{0,00} \\
\hline \multicolumn{2}{|c|}{$\begin{array}{l}\text { Neoplasias } \\
\text { selecionadas }\end{array}$} & 2223 & 39,1 & 141433 & 33,3 & & \\
\hline CID-10 & $\begin{array}{l}\text { Localização } \\
\text { anatômica }\end{array}$ & & & & & & \\
\hline 10 & Orofaringe & 242 & 10,9 & 10304 & 7,3 & 34,9 & 0,00 \\
\hline 13 & Hipofaringe & 62 & 2,8 & 2996 & 2,1 & 4,5 & 0,03 \\
\hline 15 & Esôfago & 625 & 28,1 & 39667 & 28,0 & 0,0 & 0,96 \\
\hline 32 & Laringe & 427 & 19,2 & 21771 & 15,4 & 17,3 & 0,00 \\
\hline 34 & Pulmão & 826 & 37,2 & 64580 & 45,7 & 25,3 & 0,00 \\
\hline 62 & Testículo & 41 & 1,8 & 2115 & 1,5 & 1,7 & 0,19 \\
\hline
\end{tabular}


IC95\%:1,32-1,61) e de esôfago (RCM=1,14; IC95\%:1,05-1,24) em relação à população comparada. Quanto ao câncer de pulmão, verificamos que a exposição em pintores mais jovens conferiu efeito protetor, quando comparados à população geral (RCM=0,86; IC95\%:0,75-0,86).

Na região Sudeste, pintores do sexo masculino apresentaram um aumento de risco para o câncer de orofaringe $(\mathrm{RCM}=1,58$; IC95\%:1,38-1,82) e de laringe (RCM=1,28; IC95\%:1,15-1,43), quando analisados sem estratificação por faixa etária. Ao se estratificar por grupos etários, não foram observados aumentos na magnitude do risco de morte por neoplasias específicas em

Tabela 3. Razão de chance de mortalidade (RCM) em pintores por neoplasias selecionadas, estratificada por faixa etária das regiões Sudeste e Sul do Brasil, de 1996-2013

\begin{tabular}{|c|c|c|c|c|c|}
\hline & \multicolumn{4}{|c|}{ RCM (IC95\%) } \\
\hline & & $\mathbf{N}$ & Total & $\begin{array}{l}25-44 \\
\text { anos }\end{array}$ & $\begin{array}{l}45-64 \\
\text { anos }\end{array}$ \\
\hline CID-10 & $\begin{array}{l}\text { Localização } \\
\text { anatômica }\end{array}$ & & & & \\
\hline 10 & Orofaringe & 242 & $\begin{array}{c}1,48 \\
(1,30-1,68)\end{array}$ & $\begin{array}{c}1,27 \\
(0,89-1,83)\end{array}$ & $\begin{array}{c}1,73 \\
(1,51-1,98)\end{array}$ \\
\hline 13 & Hipofaringe & 62 & $\begin{array}{c}1,30 \\
(1,01-1,67)\end{array}$ & $\begin{array}{c}0,89 \\
(0,37-2,15)\end{array}$ & $\begin{array}{c}1,56 \\
(1,20-2,03)\end{array}$ \\
\hline 15 & Esôfago & 625 & $\begin{array}{c}0,99 \\
(0,92-1,07)\end{array}$ & $\begin{array}{c}0,99 \\
(0,79-1,24)\end{array}$ & $\begin{array}{c}1,14 \\
(1,05-1,24)\end{array}$ \\
\hline 32 & Laringe & 427 & $\begin{array}{c}1,32 \\
(1,12-1,36)\end{array}$ & $\begin{array}{c}1,05 \\
(0,77-1,44)\end{array}$ & $\begin{array}{c}1,45 \\
(1,32-1,61)\end{array}$ \\
\hline 34 & Pulmão & 826 & $\begin{array}{c}0,86 \\
(0,75-0,86)\end{array}$ & $\begin{array}{c}0,82 \\
(0,66-1,03)\end{array}$ & $\begin{array}{c}0,93 \\
(0,86-0,99)\end{array}$ \\
\hline 62 & Testículo & 41 & $\begin{array}{c}1,22 \\
(0,90-1,66)\end{array}$ & $\begin{array}{c}0,95 \\
(0,68-1,34)\end{array}$ & $\begin{array}{c}1,38 \\
(0,68-2,77)\end{array}$ \\
\hline
\end{tabular}

pintores mais jovens. Para o maior grupo etário, foi observado um aumento na magnitude do risco de morte para câncer de orofaringe ( $\mathrm{RCM}=1,80$; IC95\%:1,55-2,08), de esôfago ( $\mathrm{RCM}=1,15$; IC95\%:1,04-1,27) e de laringe (RCM=1,49; IC95\%:1,33-1,67). $\mathrm{Na}$ análise da região Sul, sem estratificação etária, verificamos aumento no risco de morte em pintores para câncer de hipofaringe (RCM=1,72; IC95\%:1,16-2,53). Pintores mais velhos apresentaram um aumento no risco de morte para o câncer de orofaringe ( $\mathrm{RCM}=1,44$; IC95\%:1,03-2,01), de hipofaringe (RCM=2,19; IC95\%:1,45-3,31), de esôfago (RCM=1,18; IC95\%:1,02-1,38) e de laringe (RCM=1,36; IC95\%:1,10-168) (Tabela 4).

\section{DISCUSSÃO}

As estimativas encontradas no presente estudo sugerem aumento significativo do risco de morte por câncer de orofaringe, de laringe e de esôfago em pintores da faixa etária de 45 a 64 anos das regiões Sul e Sudeste, quando comparados à população geral. Para o câncer de nasofaringe, somente foi observado aumento do risco em pintores mais velhos da região Sul. Esses achados são corroborados por estudos de caso-controle e coorte que sustentam associações positivas entre a ocupação de pintor e o desenvolvimento de câncer no trato respiratório e esôfago ${ }^{24-26}$. Embora existam evidências epidemiológicas suficientes que sugiram aumento de risco para o câncer de pulmão em pintores ${ }^{1,3}$, nossos resultados não encontraram associação para essa ocupação com ou sem estratificação por idade e região.

Embora a literatura forneça evidências de associações positivas entre a exposição a substâncias químicas presentes na atividade laboral de pintores e alguns cânceres estudados ${ }^{27}$, o tabagismo

Tabela 4. Razão de chance de mortalidade (RCM) em pintores por neoplasias selecionadas, estratificada por faixa etária e região de residência, de 1996-2013

\section{RCM (IC95\%)}

$\begin{array}{lll}\mathrm{N} & \text { Total 25-44 }\end{array}$

\section{Região Sudeste}

\section{CID-10}

10

13

15

32

34

62

\section{Localização anatômica}

$\begin{array}{ccc}\text { Orofaringe } & 202 & 1,58(1,38-1,82) \\ \text { Hipofaringe } & 36 & 1,12(0,81-1,56) \\ \text { Esôfago } & 440 & 1,04(0,94-1,14) \\ \text { Laringe } & 325 & 1,28(1,15-1,43) \\ \text { Pulmão } & 587 & 0,87(0,80-0,94) \\ \text { Testículo } & 24 & 1,16(0,77-1,74)\end{array}$

$1,42(0,95-2,11)$

$0,54(0,13-2,16)$

$1,09(0,83-1,42)$

$0,89(0,59-1,34)$

$0,95(0,74-1,22)$

$0,83(0,51-1,34)$
45-64

\begin{tabular}{cccc}
\hline $\begin{array}{l}\text { Região Sul } \\
\text { CID-10 }\end{array}$ & $\begin{array}{c}\text { Localização anatômica } \\
\text { Orofaringe }\end{array}$ & 40 & $1,11(0,81-1,52)$ \\
10 & Hipofaringe & 26 & $1,72(1,16-2,53)$ \\
13 & Esôfago & 185 & $0,92(0,80-1,06)$ \\
15 & Laringe & 102 & $1,12(0,92-1,36)$ \\
32 & Pulmão & 239 & $0,69(0,61-0,78)$ \\
34 & Testículo & 17 & $1,36(0,84-2,20)$ \\
62 & & & \\
\hline
\end{tabular}

$\begin{array}{ll}0,84(0,35-2,03) & 1,44(1,03-2,01) \\ 1,53(0,48-4,82) & 2,19(1,45-3,31) \\ 0,78(0,51-1,19) & 1,18(1,02-1,38) \\ 1,39(0,84-2,28) & 1,36(1,10-1,68) \\ 0,55(0,34-0,87) & 0,91(0,80-1,03) \\ 1,08(0,66-1,77) & 0,49(0,07-3,51)\end{array}$

$1,80(1,55-2,08)$
$1,34(0,96-1,88)$
$1,15(1,04-1,27)$
$1,49(1,33-1,67)$
$0,97(0,89-1,05)$
$1,95(0,92-4,14)$ 
e o consumo de álcool são considerados os principais fatores de risco para câncer de pulmão, de cavidade oral, de faringe e de laringe ${ }^{28-32}$. Uma vez que o SIM não possui informações sobre esses potenciais fatores de risco, a falta de controle dessas variáveis pode produzir distorções nas estimativas de risco encontradas neste estudo.

Adicionalmente, a falta de informações na declaração de óbito relacionadas ao número de anos empregados na atividade de pintor dificulta a avaliação da relação entre a ocupação, como proxy da exposição, e a magnitude do risco de morte para os tipos de câncer estudados. Dessa forma, ocupações pontuais referentes a um período muito curto de atividade profissional como pintor não refletirão as condições de exposição, podendo levar a distorções nos resultados encontrados.

De acordo com a Organização Mundial de Saúde, 19\% dos cânceres no mundo estão relacionadas às exposições ambientais, as quais incluem o meio de trabalho, resultando em 1,3 milhão de mortes anualmente ${ }^{33}$. Considerando que o câncer ocupacional possui uma relação causa-efeito relativamente estabelecida por parte da literatura científica para algumas substâncias, estratégias para o seu controle e prevenção podem ser adotadas no ambiente de trabalho ${ }^{34}$. Embora ocorra um declínio das estimativas de diferentes tipos de câncer atribuíveis à exposição ocupacional nos países mais desenvolvidos, esse comportamento não tem sido observado em países em desenvolvimento $\mathrm{e}$ subdesenvolvidos $^{35}$. A flexibilização das leis trabalhistas e o estímulo ao desenvolvimento por meio da utilização de tecnologias ultrapassadas em países em desenvolvimento estão associadas a riscos aos trabalhadores.

O Brasil ainda não possui uma legislação específica que regule o registro e o controle de tintas, vernizes e materiais de revestimento ${ }^{36}$. Dessa forma, não existe um programa de monitoramento legal que avalie as substâncias químicas e suas respectivas concentrações presentes em tintas e vernizes ${ }^{37}$. Consequentemente, informações qualitativas e quantitativas dos constituintes de produtos do processo de trabalho de pintores somente são obtidas por meio de estudos técnicos ou científicos. Patiño Guío ${ }^{38}$ avaliou amostras de diferentes tintas à base de água produzidas e utilizadas no Brasil. Os resultados mostraram que as amostras podem emitir até 61 substâncias químicas juntas, das quais duas delas, 1,4 dioxano e etilbenzeno, possivelmente sejam carcinogênicas em humanos (grupo 2B), segundo classificação da IARC.

Outro ponto importante relacionado à deficiência de legislação específica para tintas e afins diz respeito à rotulagem. Assim, não existem exigências padronizadas para rótulos nem um órgão que fiscalize as informações disponibilizadas pelos fabricantes ${ }^{36}$. Em relação à legislação existente, a obrigatoriedade das Fichas de Informação de Segurança de Produtos Químicos (FISPQs) para produtos químicos perigosos, estabelecida pelo Decreto $\mathrm{n}^{\circ} 2.657$, de 3 de julho de $1998^{39}$, é a única garantia de informação detalhada sobre os componentes e sua periculosidade no ambiente de trabalho. Entretanto, o caráter autodeclaratório das FISPQs não garante a fidelidade e a completude das informações.

O presente estudo utilizou dados do SIM para avaliar o risco de morte por cânceres específicos entre pintores brasileiros e compará-lo ao da população geral. Essa estratégia de análise possui vantagens e desvantagens que precisam ser consideradas ao discutirmos os resultados. Por se tratar de um estudo ecológico, a utilização de dados agregados pode não refletir a associação das variáveis em nível individual, caracterizando a possibilidade de falácia ecológica. A mudança de código das ocupações no ano de 2006 pode influenciar a descrição das ocupações no SIM, por ocasionar um viés de informação, subestimando o número de casos de câncer em pintores.

\section{CONCLUSÃO}

Nossos resultados sugerem que pintores brasileiros, especialmente aqueles entre 45-64 anos de idade, apresentam maiores chances de morte por alguns cânceres do trato respiratório, como os de orofaringe, hipofaringe, esôfago e laringe.

\section{REFERÊNCIAS}

1. International Agency for Research on Cancer. Some organic solvents, resin monomers and related compounds, pigments and occupational exposures in paint manufacture and painting. Lyon: IARC; 1989. p. 1-442. (IARC Monographs on the Evaluation of Carcinogenic Risks to Humans, 44).

2. International Agency for Research on Cancer. Painting, Firefighting. Shiftwork. Lyon: IARC; 2010. p. 1-764. (IARC Monographs on the Evaluation of Carcinogenic Risks to Humans, 98)

3. International Agency for Research on Cancer. Chemical Agents and Related Occupations. Occupational Exposure as a Painter. Lyon: IARC; 2012. p. 509-39. (IARC Monographs on the Evaluation of Carcinogenic Risks to Humans, 100F)
4. Engholm G, Englund A. Cancer incidence and mortality among Swedish painters. In: Englund A, Ringen K, Mehlman MA, editors. Advances in modern environmental toxicology: occupational health hazards of solvents. New Jersey: Princeton Scientific Publishers; 1982. Vol. II, p. 173-185.

5. Gubéran E, Usel M, Raymond L, Tissot R, Sweetnam PM. Disability, mortality, and incidence of cancer among Geneva painters and electricians: A historical prospective study. Br J Ind Med. 1989;46(1):16-23. PMid:2920139.

6. Skov T, Weiner J, Pukkala E, Malker H, Andersen A, Lynge E. Risk for cancer of the pharynx and oral cavity among male painters in the Nordic countries. Arch Environ Health. 1993;48(3):176-80. PMid:8333788. http:// dx.doi.org/10.1080/00039896.1993.9940817. 
7. Guha N, Merletti F, Steenland NK, Altieri A, Cogliano V, Straif K. Lung cancer risk in painters: a meta-analysis. Cien Saude Colet. 2011;16(8):361332.

8. Wünsch-Filho V, Moncau JE, Mirabelli D, Boffetta P. Occupational risk factors of lung cancer in São Paulo, Brazil. Scand J Work Environ Health. 1998;24(2):118-24. PMid:9630059. http://dx.doi.org/10.5271/sjweh.288.

9. Andreotti M, Rodrigues AN, Cardoso LMN, Figueiredo RAO, Eluf-Neto J, Wünsch-Filho V. Ocupação e câncer da cavidade oral e orofaringe. Cad Saude Publica. 2006;22(3):543-52. PMid:16583098. http://dx.doi.org/10.1590/ S0102-311X2006000300009.

10. Ramos A, Silva Fo JF, Jardim SR. Dados sociodemográficos e condições de trabalho de pintores expostos a solventes em uma universidade. Rev Bras Saúde Ocup. 2007;32(116):38-49.

11. Dellaméa M FF, Siebel AM, Souza RM, Silva LB, Teixeira PJZ. Ocorrência de sintomas respiratórios em trabalhadores de oficinas de pintura automotiva na região do Vale do Rio dos Sinos, Rio Grande do Sul, Brasil. Acta Toxicológica Argentina. 2012;20(2):51-6.

12. Binder MCP, Wernick R, Penaloza ER, Almeida IMD. Condições de trabalho em oficinas de reparação de veículos automotores de Botucatu (São Paulo): nota prévia. Informe Epidemiol Sus. 2001;10(2):67-79. http:// dx.doi.org/10.5123/S0104-16732001000200002.

13. Neves EB, Mendonça Jr N, Moreira MFR. Avaliação da exposição a metais numa oficina de recuperação de armamento de uma organização militar. Cien Saude Colet. 2009;14(6):2269-80. PMid:20069197. http://dx.doi. org/10.1590/S1413-81232009000600036.

14. International Agency for Research on Cancer. Chromium, nickel and welding. Lyon: IARC; 1990. p. 1-648. (IARC Monographs on the Evaluation of Carcinogenic Risks to Humans, 49).

15. International Agency for Research on Cancer. Formaldehyde, 2 Butoxyethanol and 1-tert-Butoxypropan-2-ol. Lyon: IARC; 2006. p. 1-497. (IARC Monographs on the Evaluation of Carcinogenic Risks to Humans, 88).

16. Zhu CQ, Lam TH, Jiang CQ. Lymphocyte DNA damage in bus manufacturing workers. Mutat Res Genet Toxicol Environ Mutagen. 2001;491(1-2):173-81. PMid:11287310. http://dx.doi.org/10.1016/S1383-5718(01)00141-3.

17. Testa A, Festa F, Ranaldi R, Giachelia M, Tirindelli D, De Marco A, et al. A multi-biomarker analysis of DNA damage in automobile painters. Environ Mol Mutagen. 2005;46(3):182-8. PMid:16206220. http://dx.doi.org/10.1002/ em.20147.

18. Hoyos-Giraldo LS, Carvajal S, Cajas-Salazar N, Ruíz M, Sánchez-Gómez A. Chromosome aberrations in workers exposed to organic solvents: Influence of polymorphisms in xenobiotic-metabolism and DNA repair genes. Mutat Res. 2009;666(1-2):8-15. PMid:19481674. http://dx.doi. org/10.1016/j.mrfmmm.2009.03.003.

19. Silva JM, Santos-Mello R. Chromosomal aberrations in lymphocytes from car painters. Mutat Res. 1996;368(1):21-5. PMid:8637507. http://dx.doi. org/10.1016/S0165-1218(96)90036-1.

20. Oliveira HM, Dagostim GP, Silva AM, Tavares P, Rosa LAZC, Andrade VM. Occupational risk assessment of paint industry workers. Indian J Occup Environ Med. 2011;15(2):52-8. PMid:22223950. http://dx.doi. org/10.4103/0019-5278.90374.

21. Vasconcelos AMN. Qualidade das estatísticas de óbitos no Brasil: uma classificação das Unidades da Federação. In: Anais do XII Encontro de
Estudos Populacionais da Associação Brasileira de Estudos Populacionais; 2000; Campinas. Belo Horizonte: Associação Brasileira de Estudos Populacionais; 2000 [citado em 2016 Out 04]. Disponível em: http://www. abep.nepo.unicamp.br/docs/anais/pdf/2000/todos/saut7_1.pdf

22. Berenstein CK, Lacerda MA. A qualidade dos dados de mortalidade no Brasil: uma aplicação do método grade of membership (GoM) aos dados do SIM, 2002. In: Encontro Nacional de Estudos Populacionais; 2006; Caxambu. Belo Horizonte: Associação Brasileira de Estudos Populacionais; 2006. [citado em 2016 Out 04]. Disponível em: http://www.abep.nepo. unicamp.br/encontro2006/docspdf/ABEP2006_164.pdf

23. Miettinen OS, Wang JD. An alternative to the proportionate mortality ratio. Am J Epidemiol. 1981;114(1):144-8. PMid:7246522.

24. Tarvainen L, Kyyrönen P, Kauppinen T, Pukkala E. Cancer of the mouth and pharynx, occupation and exposure to chemical agents in Finland [in 1971-95]. Int J Cancer. 2008;123(3):653-9. PMid:18470913. http://dx.doi. org/10.1002/ijc.23286.

25. Pukkala E, Martinsen JI, Lynge E, Gunnarsdottir HK, Sparén P, Tryggvadottir $\mathrm{L}$, et al. Occupation and cáncer-follow-up of 15 million people in five Nordic countries. Acta Oncol (Madr). 2009;48(5):646-790. PMid:19925375. http:// dx.doi.org/10.1080/02841860902913546.

26. Bayer O, Cámara R, Zeissig SR, Ressing M, Dietz A, Locati LD, et al. Occupation and cancer of the larynx: a systematic review and meta-analysis. Eur Arch Otorhinolaryngol. 2014;273:1-12. PMid:25311307.

27. Sorahan T, Cross H, Sadhra S, Ayres J. Occupational cancer risks in commercial painters: a review prepared for the Industrial Injuries Advisory Council (IIAC). Edgbaston: Institute of Occupational and Environmental Medicine/University of Birmingham; 2010 [citado em 2016 Out 04]. Disponível em: https://www.gov.uk/government/uploads/system/uploads/ attachment_data/file/330365/review-of-cancer-painters.pdf

28. Hecht SS. Tobacco smoke carcinogens and lung cancer. J Natl Cancer Inst. 1999;91(14):1194-210. PMid:10413421. http://dx.doi.org/10.1093/ jnci/91.14.1194.

29. Dal Maso L, La Vecchia C, Polesel J, Talamini R, Levi F, Conti E, et al. Alcohol drinking outside meals and cancers of the upper aero-digestive tract. Int J Cancer. 2002;102(4):435-7. PMid:12402316. http://dx.doi.org/10.1002/ ijc. 10723.

30. Znaor A, Brennan P, Gajalakshmi V, Mathew A, Shanta V, Varghese C, et al. Independent and combined effects of tobacco smoking, chewing and alcohol drinking on the risk of oral, pharyngeal and esophageal cancers in Indian men. Int J Cancer. 2003;105(5):681-6. PMid:12740918. http:// dx.doi.org/10.1002/ijc.11114.

31. Ansary-Moghaddam A, Huxley RR, Lam TH, Woodward M. The risk of upper aero digestive tract cancer associated with smoking, with and without concurrent alcohol consumption. Mt Sinai J Med. 2009;76(4):392-403. PMid:19642154. http://dx.doi.org/10.1002/msj.20125.

32. Hsu W-L, Chien Y-C, Chiang C-J, Yang H-I, Lou P-J, Wang C-P, et al. Lifetime risk of distinct upper aerodigestive tract cancers and consumption of alcohol, betel and cigarette. Int J Cancer. 2014;135(6):1480-6. PMid:24535776. http://dx.doi.org/10.1002/ijc.28791.

33. World Health Organization. Environmental and occupational cancers. Geneva; 2011. (WHO Media Centre Fact Sheet, 350).

34. World Health Organization. Prevention of occupational cancer. Geneva; 2006. (WHO Technical Report Series, 1-16). 
35. Hashim D, Boffetta P. Occupational and environmental exposures and cancers in developing countries. Ann Glob Health. 2014;80(5):393-411. PMid:25512155. http://dx.doi.org/10.1016/j.aogh.2014.10.002.

36. Associação de Proteção ao Meio Ambiente. Câmara Técnica de Qualidade Ambiental e Gestão de Resíduos: ref.: processo no 02000.001299/201114.Voto: contrário à revisão da Resolução CONAMA. 2002 [citado em 2016 Out 04]. Disponível em: http://www.mma.gov.br/port/conama/ processos/93127174/Voto_APROMAC.pdf

37. Salasar CJ. Estudo sobre emissão de compostos orgânicos voláteis COVS em tintas imobiliárias a base de solvente e água [dissertação]. Londrina: Universidade Estadual de Londrina; 2006.
38. Patiño Guío LM. Compostos orgânicos voláteis em tintas imobiliárias: caracterização e efeitos sobre a qualidade do ar em ambientes internos construídos [dissertação]. São Carlos: Universidade de São Paulo; 2014.

39. Brasil. Decreto ${ }^{\circ} 2.657$, de 3 de julho de 1998. Promulga a Convenção $n^{\circ}$ 170 da OIT, relativa à Segurança na Utilização de Produtos Químicos no Trabalho, assinada em Genebra, em 25 de junho de 1990. Diário Oficial da União, Brasília, 06 de julho de 1998.

Recebido em: Out. 04, 2016

Aprovado em: Nov. 23, 2016 